\title{
EFFECT OF CO-TRIMOXAZOLE ON FAECAL ENTEROBACTERIA: NO EMERGENCE OF RESISTANT STRAINS
}

\author{
Ellen C. MoORhouse and WeNDy FarRell \\ Department of Clinical Microbiology, Royal College of Surgeons, Dublin 2
}

TRIMETHOPRIM-RESISTANT mutants can be isolated in the laboratory when bacterial cultures are exposed to the drug (Darrell, Garrod and Waterworth, 1968). We have investigated the effect of oral co-trimoxazole on faecal enterobacteria, looking in particular for the emergence of resistant strains.

\section{MATERIALS AND METHODS}

\section{Patients}

A total of 14 patients attending their family doctor agreed to take part in the investigation. Each was prescribed two capsules of Bactrim (Roche Ltd, Welwyn Garden City) twice daily for 5 days; a capsule contained trimethoprim $80 \mathrm{mg}$ and sulphamethoxazole $400 \mathrm{mg}$. The patients were asked to post a specimen of faeces, in the plastic container provided, to us, (i) before they started treatment, (ii) after 2 days on treatment, (iii) after 5 days on treatment, and (iv) $5 \mathrm{wk}$ after the end of treatment. None of the patients had been treated with antibacterial drugs for at least $6 \mathrm{mth}$ previously. Ages ranged from $30-50 \mathrm{yr} ; 11$ of the 14 patients were females.

\section{Bacteriological methods}

Faeces were seeded on to two MacConkey agar plates, one to obtain single colonies and the other for direct sensitivity tests with Oxoid Multodisc U1. After overnight incubation, the bacterial growth in the area surrounding any disk was purified, the colonies were identified and their resistance patterns were determined (Moorhouse and McKay, 1968).

Total counts and proportions of drug-resistant enterobacteria. Faeces were emulsfied in quarter-strength Ringer solution and ten-fold dilutions were plated on drug-free MacConkey agar and on MacConkey agar containing ( $\mu \mathrm{g}$ per $\mathrm{ml}$ ) one of the following: ampicillin (A) 25, streptomycin (S) 15, tetracycline (T) 15, chloramphenicol (C) 25, and neomycin (N) 25 .

Sensitivity to co-trimoxazole, sulphamethoxazole and trimethoprim. All strains resistant to $\mathrm{A}, \mathrm{S}, \mathrm{T}, \mathrm{C}$, or $\mathrm{N}$, and each of ten identified colonies from the single-colony plate were tested with impregnated disks for sensitivity to co-trimoxazole ( $25 \mu \mathrm{g}$ per disk), sulphamethoxazole ( $25 \mu \mathrm{g}$ per disk) and trimethoprim (1.2 $\mu \mathrm{g}$ per disk) on Oxoid Disc Sensitivity Test Agar (DST agar) with the addition of 4 per cent. lysed horse-blood (Waterworth, 1969). Zones of inhibition were compared with those produced by known sensitive and resistant Escherichia coli strains. Minimal inhibitory concentrations (MIC) for sulphamethoxazole were determined by seeding a suitable standardised inoculum of each resistant strain on sulphamethoxazole-incorporated DST-agar plates with added lysed blood. The MIC was taken as the lowest concentration to prevent the appearance of visible growth.

Resistance transfer. All resistant colonies were tested for transferable resistance by methods described previously (Moorhouse and McKay).

Additional method for the isolation of coliform bacteria from faecal specimen. When coliform bacteria were not isolated on direct plating, approximately $0.5 \mathrm{~g}$ faeces was emulsfied in $2 \mathrm{ml}$ nutrient broth. After overnight incubation, a blood-agar and a MacConkey-agar plate were flooded with the broth culture and incubated aerobically. Bacteria that grew were purified and identified. 
All cultures were incubated aerobically; no attempt was made to isolate anaerobic commensals.

\section{RESULTS \\ Effect on faecal enterobacteria}

Before treatment, E. coli or Klebsiella was isolated from the faeces of 11 of the 14 patients (the table); after 2 days' therapy, $E$. coli was isolated from the faeces of five of 14 patients; 3 days later, $E$. coli was isolated from only two of 13 patients; the 2nd day and the 5th day specimens from one patient yielded $E$. coli only after enrichment in nutrient broth. Absence of enterobacteria did not appear to be associated with diarrhoea in our patients; faecal samples were normal in colour and consistency. Five weeks after the end of treatment, $E$. coli was isolated from 12 of the 13 specimens received.

No member of the Enterobacteriaceae other than $E$. coli or Klebsiella was isolated from any specimen. A mixture of Staphylococcus albus and lactobacilli was isolated on culture from all specimens not yielding enterobacteria.

\section{Resistance patterns and transfer}

Eight"pre-treatment specimens yielded $E$. coli or Klebsiella that carried R factors transmissible to $E$. coli K12. Resistance was usually multiple and often included resistance to sulphonamides (the table). There was no significant difference in the frequency with which $\mathrm{R}+$ bacteria were found, or in the proportions of enterobacteria resistant to ampicillin, streptomycin, tetracycline, chloramphenicol or neomycin, in the faecal specimens examined before or after therapy.

\section{Sensitivity to co-trimoxazole, sulphamethoxazole and trimethoprim}

Enterobacteria resistant to co-trimoxazole or trimethoprim were not isolated from any of the faecal specimens received. Strains resistant to sulphamethoxazole were isolated from eight of the 14 pre-treatment specimens; the MIC for sulphamethoxazole was greater than $500 \mu \mathrm{g}$ per $\mathrm{ml}$ in all cases and greater than $1000 \mu \mathrm{g}$ per $\mathrm{ml}$ in strains from three of the eight specimens.

Small proportions (one of ten tested colonies) of $E$. coli resistant to sulphamethoxazole were isolated from two of five specimens after 2 days' therapy and from one of two specimens on the 5th day of treatment. Five weeks after treatment, sulphamethoxazole-resistant strains were isolated from seven of 13 specimens.

\section{Discussion}

The absence of enterobacteria from the faeces of 12 of our 14 patients during treatment confirms the observations of Näff (1971), who reported the disappearance of enterobacteria during 10 days' treatment with co-trimoxazole from the faeces of all 12 of the volunteers he investigated. Pre-treatment specimens from three of our 14 patients did not contain enterobacteria, and we consider it likely from the results that these patients had started treatment before they sent us their first specimen.

Five weeks after the end of treatment, we isolated enterobacteria from 12 of the 13 specimens received. Näff recovered enterobacteria from the faeces of his volunteers 3-12 days after the administration of co-trimoxazole was stopped. The specimen from one of our patients after 5 wk did not contain enterobacteria; in a personal communication the patient denied having had another course of the drug during these 5 wk. If the absence of enterobacteria was due to the action of a drug taken $5 \mathrm{wk}$ before, the effect of co-trimoxazole can be prolonged. The absence of enterobacteria during therapy was not associated with diarrhoea; Brumfitt and Pursell (1972) reported the occurrence of diarrhoea in only one of 83 patients treated with co-trimoxazole.

The disappearance of enterobacteria from the faeces of the majority of patients appeared to be due to the action of trimethoprim alone, because their pre-treatment specimens contained sulphonamide-resistant strains, some of which were highly resistant. 


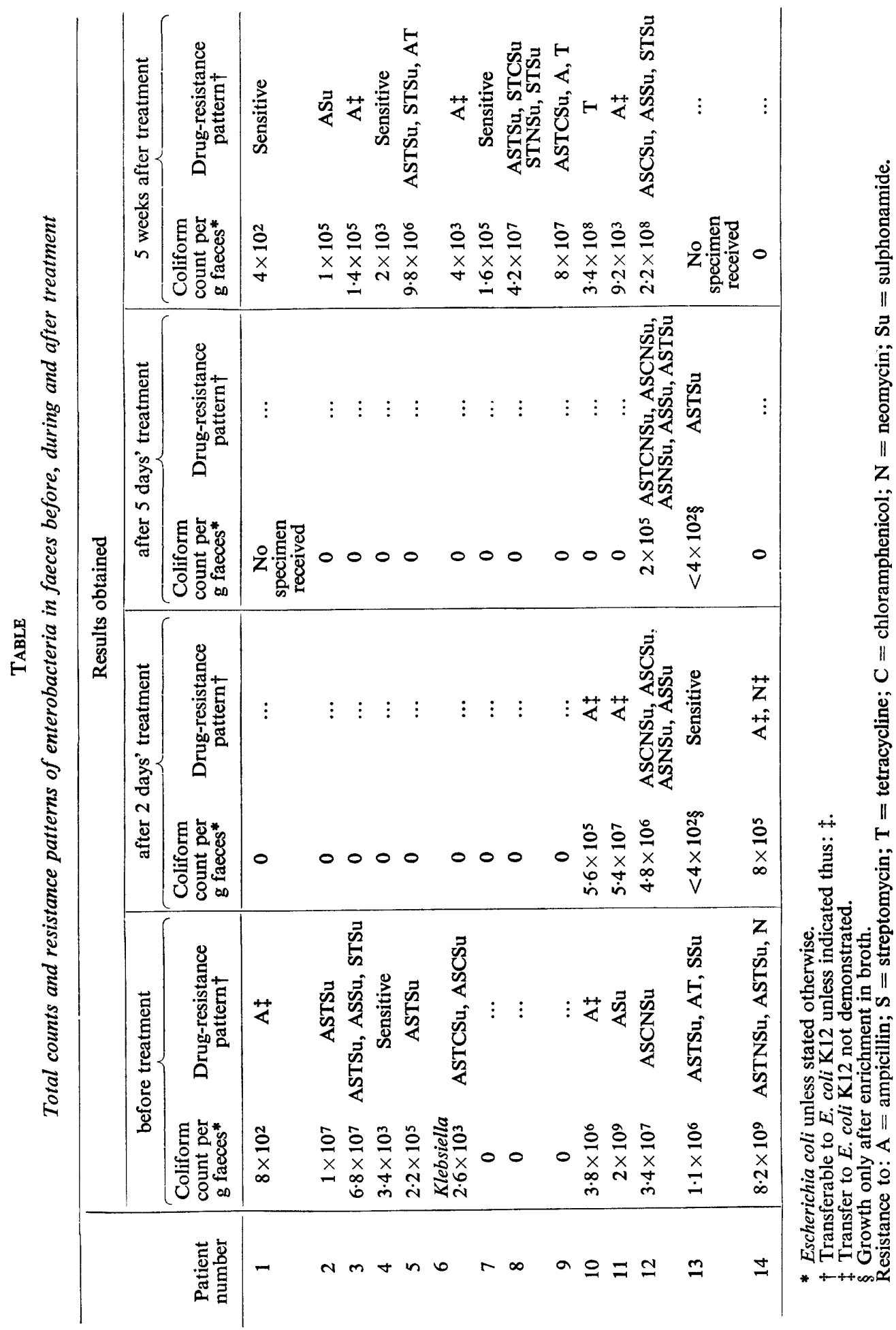


The persistence of enterobacteria in the stools of one patient throughout treatment could not be explained by the presence of strains with very high resistance to sulphonamide, because the MIC values for sulphamethoxazole in their strains were less than or similar to those found in strains isolated from other patients.

Enterobacteria resistant to co-trimoxazole or trimethoprim were not isolated during or after treatment, indicating that trimethoprim resistance acquired by mutation does not readily emerge as a result of the oral administration of co-trimoxazole. Datta et al. (1971) showed that oral tetracycline had a marked effect in converting the $E$. coli population of the bowel to tetracycline resistance and indeed to multiple-drug resistance. No such effect was found with co-trimoxazole in spite of the fact that sulphonamide-resistant bacteria were present in the majority of the faecal specimens before treatment and that sulphonamide resistance was carried by $R$ factors. $R$ factors that determine trimethoprim resistance have been demonstrated (Fleming, Datta and Grüneberg, 1972), but the fact that trimethoprimresistant bacteria were not isolated during this investigation suggests that they are not yet widely distributed.

\section{SUMMARY}

During treatment with co-trimoxazole, enterobacteria disappeared from the faeces of 12 of 14 patients investigated. Strains resistant to co-trimoxazole or trimethoprim were not isolated from any of the patients during the entire investigation.

We thank our colleagues in general practice, Dr Helen Kilgallen-O'Connell, Dr C. Dempsey, and Dr T. Shaw, for their kind collaboration and Dr J. S. Doyle, Consultant Physician, St Laurence's Hospital, Dublin, for his cooperation. Bactrim was supplied free by Roche (Products) Ltd.

This work was supported by a grant from the Medical Research Council of Ireland.

\section{REFERENCES}

BRUmfitt, W., AND PuRsell, R. 1972. Double-blind trial to compare ampicillin, cephalexin, co-trimoxazole, and trimethoprim in treatment of urinary infection. Br. Med.J., 2, 673.

Darrell, J. H., Garrod, L. P., and Waterworth, Pamela M. 1968. Trimethoprim: laboratory and clinical studies. J. Clin. Path., 21, 202.

Datta Naomi, Faiers, Mary C., Reeves, D. S., Brumfitt, W., Ørskov, F., and Ørskov, IDA 1971. $\mathrm{R}$ factors in Escherichia coli in faeces after oral chemotherapy in general practice. Lancet, $1,312$.

Fleming, M. P., Datta, Naomi, and Grüneberg, R. N. 1972. Trimethoprim resistance determined by $\mathrm{R}$ factors. Br. Med.J., 1, 726.

MoORHOUSe, Ellen C., AND MCKAY, L. 1968. Hospital study of transferable drug resistance. Br. Med. J., 2, 741.

NÄFF, H. 1971. UUber die Veränderungen der normalen Darmflora des Menschen durch Bactrim. Pathologia Microbiol., 37, 1.

Waterworth, Pamela M. 1969. Practical aspects of testing sensitivity to trimethoprim and sulphonamide. Post-grad. Med. J., 45, Suppl., p. 21. 